CERN-Data Handling Division $\mathrm{DD} / 80 / 22$

October 1980

D.A. Jacobs

APPLICATIONS OF ESOP, A FAST MICROPROGRAMMABLE PROCESSOR,

IN HIGH ENERGY PHYSICS EXPERIMENTS AT CERN

Presented at the Europhysics Conference on Computing in

High Energy and Nuclear Physics, Bologna, Italy, $9-12$

September, 1980 .

$\mathrm{DD}-\mathrm{jt}$ 


\title{
APPLICATIONS OF ESOP, A FAST MICROPROGRAMMABLE PROCESSOR, IN HIGH ENERGY PHYSICS EXPERIMENTS AT CERN.
}

David A. Jacobs.

CERN, DD Division, CH-1211 Geneva 23, Switzerland.

\begin{abstract}
ESOP is a fast 16 bit processor designed at CERN. It derives its speed from harware parallelism and from microprogramming of the application software. It has been specially developed for the applications of secondary trigger calculation and data reduction in experiments, with interfaces to several widely used detector readout systems and special processor hardware. This report summarises the work done by several groups to use ESOP in three experiments currently running at CERN.
\end{abstract}




\section{Introduction.}

ESOP is a fast microprogrammable processor, designed in DD division at CERN, which is being used for secondary trigger calculations and data restructuring in high energy physics experiments. This report reflects the work of many people both at CERN and other institutions in designing the processor and adapting it to the various applications. Its aim is to summarise the available hardware and software and to show by example the manner in which ESOP is used.

\section{Application area of ESOP.}

Present applications of the machine fall into two main categories; second level triggering and data reduction. The first is of use where the primary trigger based on logical combinations of detector signals is inadequately selective. This is by no means uncommon as physicists seek to use rather restrictive triggers to study specific interactions. In such cases it may be possible to achieve a substantial reduction in the unwanted background by performing simple calculations on a subset of the data from the detectors. This at least reduces the amount of useless data to be handled offline. If in addition the decision to reject an event can be made quickly enough, the deadtime of the experiment may be reduced by aborting or never starting the main readout. A reduction in deadtime can improve the experiment's sensitivity, implying more efficient use of the expensive particle accelerators. A reduction or compression of the data from individual detectors has the same effect of reducing deadtime and data handling load. In some cases the latter may be reduced further by using the processor to reformat the data.

ESOP has been designed to carry out these operations in the common situation that highest possible speed is required and that integer arithmetic alone is sufficient. The processor is normally placed between the detectors of the experiment and the data acquisition computer to take advantage of its high input data rate and fast response time and to allow selective, autonomous readout of the detectors. The data acquisition computer acts as a host for ESOP.

\section{Processor structure.}

The hardware of the ESOP processor has already been described in some detail [I] and only a brief outline will be given here. The machine uses 48 bit microcoded instruction words and the present version has memory space for $1 \mathrm{~K}$ instructions. One instruction is executed per machine cycle (125nsec.). It performs fixed point arithmetic on 16 bit data stored in a separate data memory. This memory may be up to $64 \mathrm{Kw}$ in total size and is built in 4 or $8 \mathrm{Kw}$ modules. Speed is gained through a high degree of parallelism. There are separate arithmetic units (ALUs) for calculation of instruction address, data address and data, and all may be used simultaneousiy. 
This can be done most effectively by microcoding the application programs. While such microcoding undoubtedly requires some programming skill from the user, an assembler language was developed (D. Myers, 1975, private communication) which much eases the task.

Zero-overhead loop control is made possible by a hardware loop counter. The arithmetic power has been increased by the addition of special processing units in parallel to the normal data $A L U$, such as multiply with 32 bit result ( 2 cycles) and shift (any number of bits in 1 cycle). More specialised extensions are the trigger-processing functions of 'loose comparison' which compares IA-BI with a limit E (2 cycles) and bit search which returns the bit numbers of bits set in a word ( 1 cycle/bit).

To give a feeling for what is involved in ESOP programming a short sequence of instructions is shown in Table 1. This example illustrates the grouping of micro-operations into instructions (delimited by .NEXT) and the last instruction shows the way in which normally sequential actions can be carried out in parallel in a pipelined fashion.

4. System Structure.

\subsection{Processor and connection to host computer.}

Table 2 summarises the ESOP hardware which is available. The processor is built in CIM modules connected by a special backplane. Each data memory module has 4 independant ports with full addressing logic which may be set up by ESOP or by the host. Two ports are available on a front-panel connector for connection of external devices, the third allows access to the memory from the host computer via a CAMAC interface and the fourth is used by ESOP itself. The processor is controlled by a second CAMAC interface which also gives access to the instruction memory. The interfaces allow block transfers using normal CAMAC DMA controllers.

\subsection{Connection to detector systems.}

External devices may be connected to ESOP either via data memory ports or a general purpose $1 / 0$ module [2]. This $1 / 0$ module has been used to give ESOP full control of a CAMAC branch via the Auxiliary Branch Controller ( $A B C$ ) module [3].

In the field where ESOP is used, the provision of fast data readout from the detectors is very important. For instance, a typical trigger calculation might take only $100 \mathrm{microseconds}$ but be based on 100 words of data which, using a CAMAC branch system, could take more than $200 \mathrm{microseconds}$ to read. For this reason, several detector readout systems have been interfaced directly to data memory ports. Since each memory module has its own ports, multiple simultaneous transfers are possible. 
4.2.1. RMH Multiwire Proportional Chamber readout system [4].

This is interfaced via a special module (J.-P. Melot, 1978 , private communication). Data transfer is externaliy triggered and the transfer rate is 4-5 words/microsecond. The RMH system can be reset automaticaliy at the end of transfer, minimising detector deadtime.

\subsubsection{ROMULUS/REMUS [5].}

A bi-directional adaptor, controlled by the $I / 0$ module, has been designed $[6]$.

\subsubsection{DTRC buffered Drift Chamber readout system.}

The branch controller of this system for buffered high speed readout of the DTR Drift Time Recording modules, developed for the European Hybrid Spectrometer (EHS) project by C. Ljuslin, has been designed from the start for direct connection to the ESOP data memory as well as CAMAC.

In addition to these general-purpose data collection systems, some special detectors have been interfaced directly to the ESOP data memory.

5. Software support.

The assembly language mentioned earlier has been implemented as a cross-assembler with macro facilities (A. Gautschi and D. Myers 1976, private communication) and to this has been added an ESOP simulator (W. Jank 1978, private communication). BCPL and FORTRAN (J. Hooper 1979, private communication) versions exist, running at present on DECsystem-10, NORD-10 (or 100), IBM 370 and UNIVAC 1100 computers. The simulator has proved a most useful tool for software development since not only does it make one independant of the availability of a real ESOP but also supports many debugging features not found on the real machine. Breakpoints may be used, instruction execution traced and timed, and all details of processor state examined and modified between cycles. For the hosts to which ESOP is attached (currently PDP-11 and NORD-10), there is a utility program in FORTRAN allowing downline loading, dumping and control, and also libraries of routines to support ESOP from the real time programs of data-acquisition systems. Finaliy, there is a set of diagnostic software running both in ESOP and the host.

6. Present applications.

ESOPs are currently in use in three experiments at CERN; NA11 [7], R807 [8] and EHS [9]. Short examinations of these applications will serve to show the areas where such a processor is of interest. 
6.1. NA11.

This experiment was the first to employ ESOP. Full details of their trigger may be found in [10]. Here only a short summary will be given. The primary trigger is on a single electron from the leptonic decay of a charm particle. High momentum hadrons and electron pairs produce the undesirable background. Two ESOPs are used, one reading wire chambers and pattern units via the RMH system for momentum measurement of the trigger particle and electron pair detection. The other reads pulse heights from an electron calorimeter $v i a$ CAMAC and the $A B C$. The experiment sensitivity has been increased by a factor of two and the data handling reduced by a further factor of two.

\subsection{R8B7.}

This experiment at the CERN Intersecting storage Rings seeks at present to trigger on observation of a single high momentum hadron emerging from the interaction region over rather a large solid angle (1 steradian). The algorithm used for secondary triggering is explained in [11]. A first selection is done by hardwired methods using coincidences between bands of two wire chambers, but uncertainties in particle direction and confusion from other particles result in more than 99 percent of false triggers. The experiment relies heavily on ESOP to reduce this background by finding candidate tracks in data from a set of cylindrical drift chambers and measuring their momentum from their curvature in a magnetic field.

A simplified block diagram of the readout equipment is shown in Fig. 1. The system is of special interest since the pre-trigger information is used to select for readout to ESOP only the DTR modules for the segments of the drift chambers through which the candidate should have passed. The selection is done by specially modified crate controllers (DECs) [12]. The data is read to ESOP $v i a$ the ROMULUS adaptor. If the pre-trigger is accepted by ESOP, the main readout of all the detectors is started. Since DTR modules may only be read once, the data transfered to ESOP is read back out onto the ROMULUS bus and merged with the data from the other DTR modules. The average time for ESOP to reject a pre-trigger is about 120 microseconds while the time to read an entire event to the data acquisition computer is some $30 \mathrm{milliseconds.} \mathrm{The} \mathrm{sensitivity} \mathrm{of}$ the experiment is increased by a factor of between 200 and 750 compared with the pre-trigger.

\subsection{EHS.}

The layout of some of the detectors of this large hybrid spectrometer is shown in Fig 2. The facility will be used for many experiments with different triggering needs and so the flexibility of a computed second level trigger is especially useful. An ESOP is installed for this purpose, reading the data from the wire chambers 
via the RMH system. For the NA16 experiment earlier this year it was programmed to compute a simple secondary particle multiplicity trigger using data from W2. The typical time for rejection was 15 microseconds.

A basic trigger problem common to most EHS experiments will be that of rejecting interactions occuring outside the useful visible volume of the bubble chamber. This so-called fiducial-volume trigger decision must be made fast enough ( $<1 \mathrm{millisec.)}$ to inhibit the taking of a picture. For 1981, ESOP will be programmed to perform this function. There are two likely methods. In the first (see Fig 3a.) the 'envelope' of secondary tracks seen by $W 1$ and $W 2$ would be projected back to determine the interaction position. In the second (see Fig 3b.) optical diode arrays would record track positions in the chamber using a special flash some time before the main one [13]. In the simplest algorithm, ESOP would use data from $U 1$ and U3 to predict the position of the candidate beam track at the positions of the arrays. Absence of a hit on the array at that point would indicate that an interaction had occured upstream.

A further use for this ESOP, already programmed for NA16, is to provide low deadtime readout of the RMH system to record the non-interacting beam particles preceding the interaction within the 0.5 milliseconds sensitive time of the bubble chamber. Such data is useful for spectrometer calibration. Deadtimes of about 11 microseconds per beam particle were achieved.

A second ESOP is installed to compress and reformat the data from the large-volume drift chamber ISIS [14]. This detector has a very high input data rate of up to 10,000 charge samples in 50 microseconds. These charges are temporarily stored in a bank of capacitors but must be read out and converted before significant leakage takes place. This is achieved in about $2 \mathrm{milliseconds}$ by four ADC channels reading in parallel to four ESOP data memory modules. To maintain accuracy of charge measurement all capacitors are always read out whether or not they were used in the 50 microseconds window. ESOP will restructure the data and remove the useless information, giving a significant reduction in later data handling.

A third ESOP is forseen for use with the drift chamber readout to perform stop-time subtraction and to re-order the data in a convenient way for offiine processing.

In addition to these experiments, there are firm plans for two more applications using the RMH readout system. Clearly this system is popular because of its high transfer rate, and it is being extended beyond its original use for proportional chambers. Pattern units exist and modules for high-speed ADCs are being planned. 
7. Conclusions.

Experience with ESOP allows a number of conclusions to be drawn. -Using only integer arithmetic, effective trigger algorithms can be written, executing at least an order of magnitude faster than in a conventional mini-computer.

-With the aid of a simulator, such algorithms have been developed without problem within the normal planning and set-up times of experiments.

- Physicists from the experimental groups can learn to produce effective microcode in a relatively short time and can then write and maintain their own application code.

-In practice, most of the instructions of trigger programs have been found to belong to time-critical loops. This fact is of importance in considering the advantages of adding a more easily programmed but slower conventional instruction set on top of the micro-code machine. It would seem difficult to make extensive use of such instructions Without significant loss of performance for the application.

- It is important to provide for the needs of triggering at the stage of detector design so as to produce as much numerical simplification as possible. 
Acknowledgements.

The main credit for ESOP must go to its hardware designers at CERN, in particular T. Lingjaerde, D. Marland and A. Fucci, and to $W$. Jank and $D$. Myers for the assembler and simulator software. Thanks must be given to the teams of experiments NAL1 and R807 for their work on software and detector interfacing and for many helpful suggestions for processor improvement. Their contribution was crucial for the development of the processor. In this respect one must especially mention D. Giddings, B. Heck, J. Hooper, G. Lutjens and S. 0 . $\mathrm{Nielsen.}$ 


\section{References.}

1. Lingjaerde T., CERN - Data Handling Division, DD/75/17, May 1975.

Lingjaerde T., CERN - Data Handling Division, DD/76/9, May 1976.

Lingjaerde T. and Marland D., CERN - Data Handling Division, DD/77/8, June 1977.

Marland D., CERN - Data Handling Division, DD/78/13, August 1978.

2. Lingjaerde T., ESOP Application Note No.1, CERN - Data Handling Division, January 1979.

3. Vanuxem J-P., ABC: An Auxiliary Branch Controller for CAMAC, EP-Electronics Note 80-06, September 1980.

4. Lindsay et al., Nucl. Inst, and Meth. 156(1978) 329-333.

5. Ponting $P$. J., A Guide to ROMULUS/REMUS data acquisition Systems, EP-Electronics Note 80-01, February 1980.

6. Cairanti S. and Heck B., A bi-directional Interface between ESOP and ROMULUS/REMUS, CERN/EF/R807-ESOP, 80-1, JUlY 1980 .

7. Blanar G. et al., CERN SPSC/78-14/P-95.

8. Albrow M. et al., CERN ISRC/76-36/P-95.

9. Allison W. W. M. et al., CERN SPSC/75-15/P-42.

10. Damerell C. et al., Presented at the Europhysics Conference on COMPUTING IN HIGH ENERGY AND NUCLEAR PHYSICS, Bologna, Italy, September $9-12,1980$.

11. Heck B. et al., Presented at the Europhysics Conference on COMPUTING IN HIGH ENERGY AND NUCLEAR PHYSICS, Bologna, Italy, September 9-12, 1980.

12. Cairanti S., Di Tore G, and Heck B.. The selective readout system for ESOP. - CERN/EF/R807-ESOP, 80-2, July 1980.

13. Anders H., CERN/EP/EHS/PH 80-2, 103-114, March 1980.

14. Allison W. W. M. et al., Nucl. Inst. and Meth. 119(1974) 499. 
Figure Captions.

1. Simplified block diagram of installation of ESOP in readout system of experiment R807.

2. Layout of part of EHS spectrometer.

U1, U2, W1, W2 - Proportional chambers.

RCBC

- Bubble chamber

D1, D2, D3, D4 - Drift chambers.

ISIS 2

- Large-volume drift chamber.

3. Proposals for fiducial volume trigger for EHS:

a) using 'envelope' of secondary tracks seen in proportional chambers $W 1$ and $W 2$.

b) using track positions as seen by two optical diode arrays looking into the bubble chamber. 


\section{CONTENTS}

1. Introduction.

2. Application area of ESOP.

3. Processor structure.

4. System Structure.

4.1 Processor and connection to host computer.

4.2 Connection to detector systems.

4.2.1 RMH Multiwire Proportional Chamber readout system

[4].

4.2 .2 ROMULUS/REMUS [5].

4.2.3 DTRC buffered Drift Chamber readout system.

5. Software support.

6. Present applications.

7. Conclusions. 


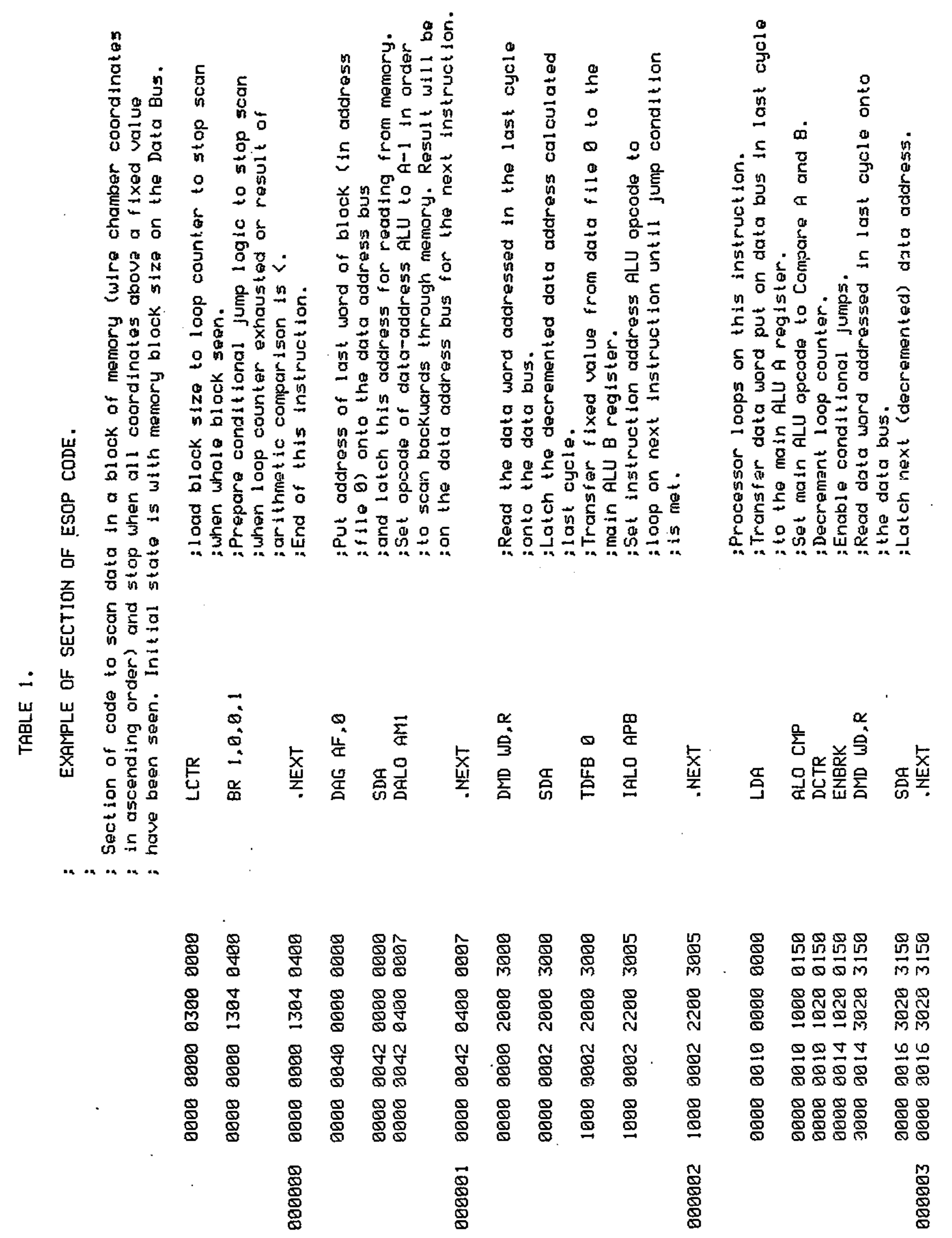

NM 
List of ESOP modules as of September 1979.

1. IM256: Instruction unit with 256 words $x 48$ bits. Speed: The processor cycle time is 125 nanoseconds, limited by the arithmetic unit.

2. IMIK: As above, but size expanded to $1 \mathrm{~K} \times 48$ bits (alternative to 1 ).

3. ALU II: Arithmetic Logic Unit with $8 \times 16$ bits register file. operations: ADD, SUB, BOOLEANS : Total 32 different operations, each taking one cycle. Fixed point multiply $(16 \times 16->32$ bits in 2 cycles). LOOSE COMPARISON: Conditional jump or skip on $1 A-B 1<=E$ in 2 cycles.

4. DM: Multi-port Data Memory $8 K \times 16$ bits. Access time from CPU: $45 n s$. DMA transfer rate: 1 word/100 ns. Up to 8 modules can work in parallel, giving a transfer rate of 80 × 16 bit words per microsec.

5. DM CONTR: Data Memory Controller with $8 \times 16$ bits address file and dedicated Arithmetic Logic Unit for address calculations operating concurrently with the rest of the processor.

6. DMA CONTR: 4 port DMA controller, one port dedicated to CAMAC. Includes arbiter and timing logic for autonomous block transfers. The transfer of an $8 \mathrm{KW}$ block can run independantly of and concurrently with the CPU activity. Address calculations and wordcount check done internally. Parameters can be set either via CAMAC or ESOP.

7. SHIFT \& SEARCH: Bit shift and bit search unit. SHIFT: Any number of shifts of any kind within one cycle. (LOGIC, ARITH. Or ROTATE, LEFT or RIGHT). BIT SEARCH: Returns the bit numbers of "ONES" in a word at the rate of one per cycle.

8. I/O and INTERRUPT: Contains $3 \times 16$ bits control 1 ines and one handshake for general purpose interfacing. Two 8 level priority interrupt ports can be used separately or in cascade. The interrupt logic works in conjunction with the Instruction Unit.

9. CAMAC INTERFACE: There are two different CAMAC interfaces: One for ESOP control and one for the DM. These units plug into any slot in a CAMAC crate. 


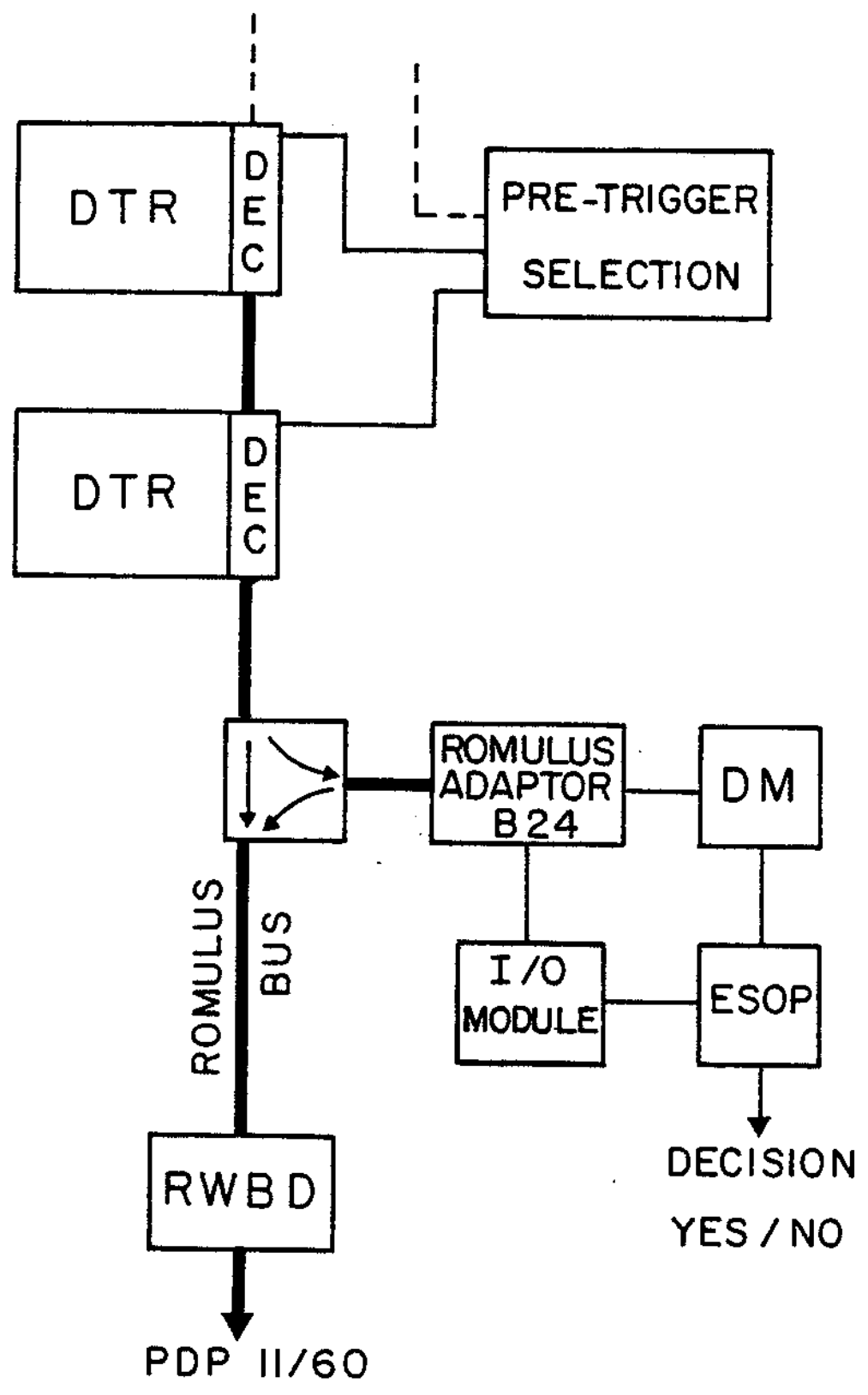

Fig. 1 


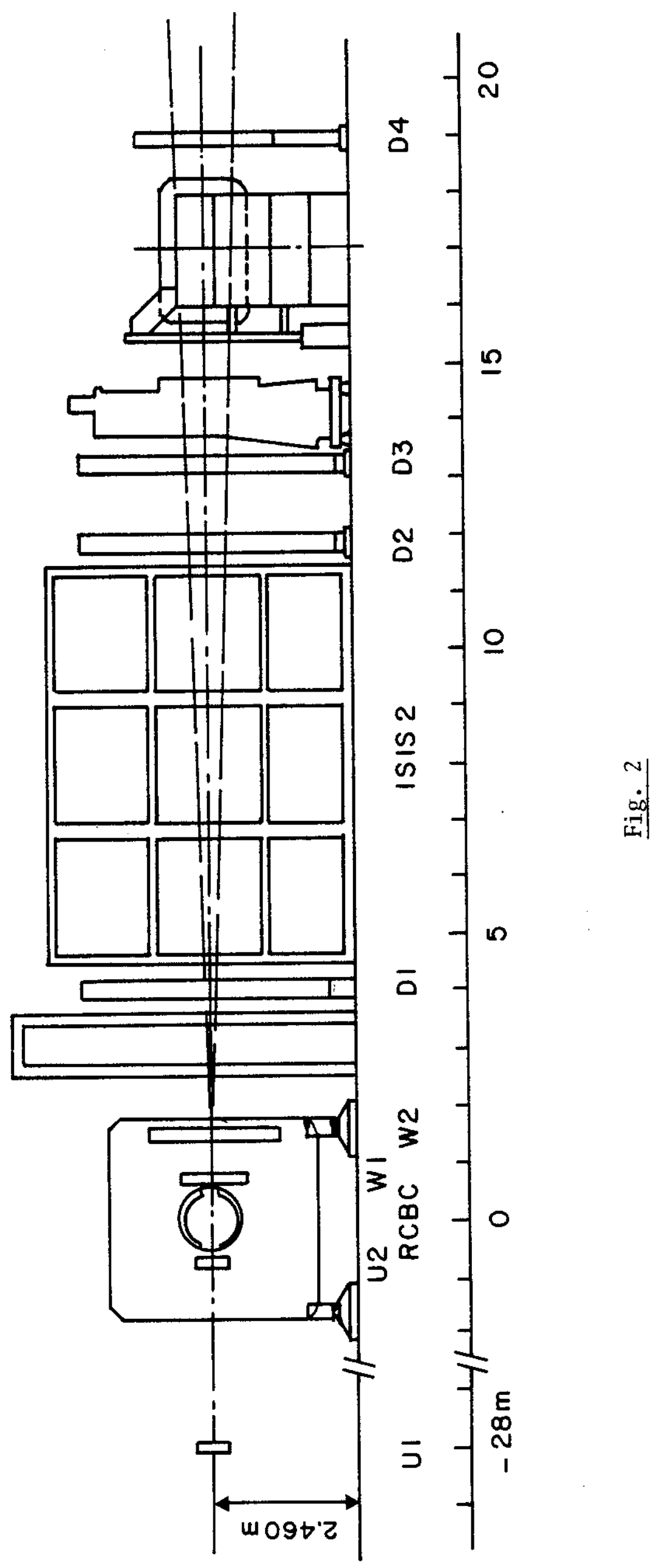




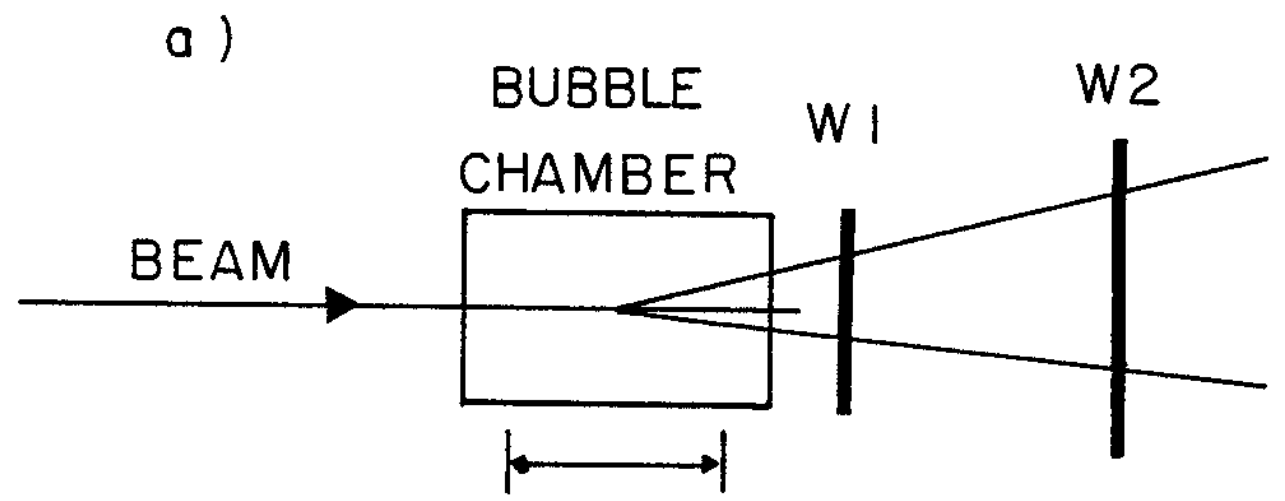

FIDUCIAL

VOLUME

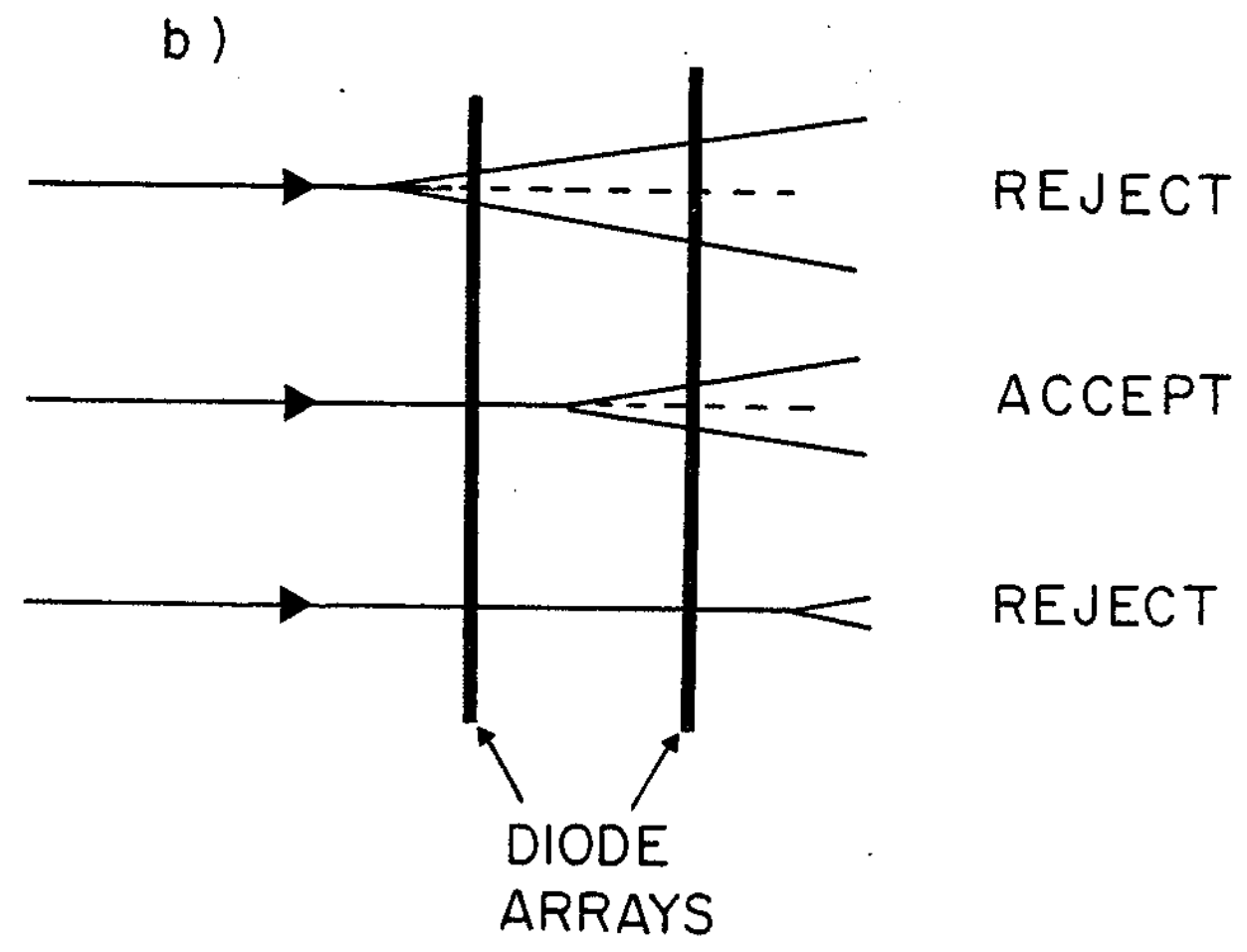

Fig. 3 
18 